H. Fujimoto.

Nagoya Math. J.

Vol. 44 (1971), 1-15

\title{
RIEMANN DOMAINS WITH BOUNDARY OF GAPAGITY ZERO
}

\author{
HIROTAKA FUJIMOTO
}

\section{§1. Introduction.}

The well-known Thullen-Remmert-Stein's theorem ([9], [7]) asserts that, for a domain $D$ in $C^{N}$ and an $n$-dimensional irreducible analytic set $S$ in $D$, a purely $n$-dimensional analytic set $A$ in $D-S$ has an essential singularity at any point in $S$ if $A$ has at least one essential singularity in $S$. In [1], E. Bishop generalized this to the case that $A$ has the boundary of capacity zero in his sense. Afterwards, in [8], W. Rothstein obtained more precise informations on the essential singularities of $A$ under the assumption $\operatorname{dim} A=1$. The main purpose in this paper is to generalize these Rothstein's results to the case of arbitrary dimensional analytic sets.

We consider a Riemann domain $(X, \pi, M)$ with boundary of capacity zero, namely, a triple of a connected $n$-dimensional normal complex space $X$, a connected $n$-dimensional complex manifold $M$ and a discrete holomorphic map $\pi: X \rightarrow M$ with the following properties:

For any $z_{0} \in M$ there are a neighborhood $U$ of $z_{0}$ and a plurisubharmonic function $u(x)$ on $\pi^{-1}(U)$ such that (i) $u(x) \leqq 0$, (ii) $u(x) \not \equiv-\infty$ on any connected component of $\pi^{-1}(U)$ and (iii) $\lim _{\nu \rightarrow \infty} u\left(x_{\nu}\right)=-\infty$ for any sequence $\left\{x_{\nu}\right\}$ without accumulation points in $X$ if $\lim _{\nu \rightarrow \infty} \pi\left(x_{\nu}\right)$ exists in $U$.

The first main result is the following

Theorem I. If $(X, \pi, M)$ is a Riemann domain with boundary of capacity zero, then $M-\pi(X)$ is of capacity zero (c.f. Definition 2.5).

We define a direct boundary point of a Riemann domain on the analogy of a direct transcendental singularity in the theory of functions of one complex variable (c.f. Definition 4.3). As an application of Theorem I, we give the following theorem, which is a generalization of a result in [4].

Received November 18, 1970 
Theorem II. The projection image of the set of all direct boundary points of a Riemann domain with boundary of capacity zero is a set of capacity zero in the base space.

Using Theorem II, we prove Rothstein's results in [8] without the assumption of dimension one. The following theorem is shown.

Theorem III. Let $D=D_{1} \times D_{2}$ be a domain in $\boldsymbol{C}^{n+k}\left(D_{1} \subset \boldsymbol{C}^{n}, D_{2} \Subset \boldsymbol{C}^{k}\right)$ and $S$ be a closed subset of $C^{n+k}(S \subset \bar{D})$. If an irreducible $n$-dimensional analytic set $A$ in $D-S$ satisfies the conditions (i) there is a plurisubharmonic function $u(x)$ on $A$ such that $0 \geqq u(x) \not \equiv-\infty$ and $\lim _{x \rightarrow S} u(x)=-\infty$, (ii) $\bar{A} \cap\left(D_{1} \times \partial D_{2}\right) \subset S$ and (iii) there is a set $P$ of positive capacity in $D_{1}$ such that each $\{z=c\} \cap A(c \in P)$ is finite, then $\bar{A} \cap\left(D_{1} \times \boldsymbol{C}^{k}\right)$ is analytic in $D_{1} \times \boldsymbol{C}^{k}$.

In this connection, we generalize the well-known Iversen's theorem to the case of Riemann domains with boundary of capacity zero and give some other applications.

\section{§2. Preliminaries on plurisubharmonic functions.}

In this paper a complex space is always assumed to be normal unless stated to the contrary. Moreover, we assume that all complex spaces and complex manifolds are $\sigma$-compact and connected.

Let $X$ be a complex space and $u(x)$ be an extended real-valued function on $X$ which permits the value $-\infty$ but not $+\infty$.

Definition 2.1. $u(x)$ is said to be plurisubharmonic on $X$ if (i) $u(x)$ is upper semi-continuous on $X$ and (ii), for any open set $W$ in the complex plane $\boldsymbol{C}$ and a holomorphic map $\phi: W \rightarrow C$, the composite $u \cdot \psi: W \rightarrow X$ is subharmonic on $W$ in the usual sense or identically equal to $-\infty$.

As is easily seen, it holds that

(2.2) (i) Let $\psi$ be a holomorphic map of $X$ into another complex space $Y$. If $u(x)$ is plurisubharmonic on $Y$, then $u \cdot \psi$ is also plurisubharmonic.

(ii) If $u(x)$ and $v(x)$ are plurisubharmonic on $X$ and $c$ is a positive real constant, then $c u, u+v$ and $\max (u, v)$ are also plurisubharmonic.

The following assertion was proved by $\mathrm{H}$. Grauert and R. Remmert in [2], Satz 3, p. 181. 
(2.3) Let $S$ be a thin analytic set in a complex space $X$ and $u(x)$ a plurisubharmonic function on $X-S$ which is bounded above. Then there exists exactly one plurisubharmonic function $\tilde{u}(x)$ on $X$ such that $\tilde{u}(x)=u(x)$ on $X-S$ and it is given by $\tilde{u}(x):=\varlimsup_{x^{\prime} \rightarrow x, x^{\prime} \in X-S} u\left(x^{\prime}\right)(x \in X)$.

The assertion (2.3) implies the following

(2.4) If $u(x)$ is a plurisubharmonic function on $X$ and $S$ is a thin analytic subset of $X$, then we have $u(x)=\varlimsup_{x^{\prime} \rightarrow x, x \in X-S} u\left(x^{\prime}\right)$ for any $x \in X$.

Now, we give the definition of a subset of capacity zero in a complex space $X$ (c.f. T. Nishino [6], p. 232).

Definition 2.5. We shall say a subset $S$ of $X$ to be of capacity zero in $X$ and write it $\operatorname{cap}(S)=0$ if we can take a countable family $\left\{S_{\nu}\right\}$ of subsets of $X$ such that $S=\cup_{\nu} S_{\nu}$ and, for each $S_{\nu}$, there exists a plurisubharmonic function $u_{\nu}$ on a connected open set $U_{\nu}$ satisfying the conditions that (i) $u_{\nu}(x) \not \equiv-\infty$ on $U_{\nu}$ and (ii) $S_{\nu} \subset\left\{x \in U_{\nu} ; u_{\nu}(x)=-\infty\right\}$. If $S$ is not of capacity zero, it is said to be a set of positive capacity and denoted by $\operatorname{cap}(S)>0$.

REmark. A closed set in the complex plane $\boldsymbol{C}$ is of capacity zero in the sense of Definition 2.5 if and only if it is of logarithmic capacity zero in the usual sense.

Easily, we have

(2.6) (i) If each $S_{\nu}(\nu=1,2, \cdots)$ is of capacity zero, so is the union $\cup_{\nu} S_{\nu}$.

(ii) Any subset of a set of capacity zero is of capacity zero.

(iii) If $S$ is of positive capacity, the set

$S^{\prime}:=\{x \in S ; \operatorname{cap}(S \cap U)>0$ for any neighborhood $U$ of $x\}$

is also of positive capacity.

\section{$\S 3$. A generalization of the Riemann theorem on removable sigularities.}

For later use, we shall prove

Proposition 3.1. Let $X$ be a complex space and $S$ be a closed subset of capacity zero in $X$. If a holomorphic function $f$ on $X-S$ is locally bounded on $S$, i.e., bounded on some neighborhood of each $x \in S$, then it has exactly one holomorphic continuation to $X$. 
Proof. Firstly, under the assumption that $X$ is a complex manifold, we shall show that, for each $x_{0} \in S, f$ has a holomorphic continuation to a neighborhood of $x_{0}$. Take a sufficiently small neighborhood $V$ of $x_{0}$ which can be written $V=\left\{\left|z_{i}\right|<1,1 \leqq i \leqq n\right\}$ with a system of local coordinates $z_{1}, \cdots, z_{n}$ defined on some neighborhood of $\bar{V}$. There is no harm in assuming that $S \subset V$ and $S=\cup_{\nu} S_{\nu}$, where each $S_{\nu}$ is included in $\left\{x \in U_{\nu} ; u_{\nu}(x)\right.$ $=-\infty\}$ for a suitable plurisubharmonic function $u_{\nu}(\not \equiv-\infty)$ on a connected open subset $U_{\nu}$ of $V$.

Let $\left\{a^{(\kappa)} ; \kappa=0,1,2, \cdots\right\}\left(a^{(\kappa)} \neq 0\right)$ and $\left\{\underline{b}^{(\mu)}:=\left(b_{2}^{(\mu)}, \cdots, b_{n}^{(\mu)}\right) ; \mu=0,1,2, \cdots\right\}$ be countable dense subsets of the sets $\left\{\left|w_{1}\right|<1 / 2\right\}$ in $C$ and $\left\{\left|w_{i}\right|<1 / 2\right.$, $2 \leqq i \leqq n\}$ in $C^{n-1}$ respectively, where we let $\underline{b}^{(0)}:=(0, \cdots, 0)$. By $\Phi_{\kappa \mu}$ we denote the non-singular linear transformation defined as follows;

$$
\begin{aligned}
z_{1} & =z_{1}^{\prime} a^{(\kappa)} \\
z_{2} & =z_{2}^{\prime} a^{(\kappa)}+b_{2}^{(\mu)} \\
& \ldots \\
z_{n} & =z_{n}^{\prime} a^{(\kappa)}+b_{n}^{(\mu)} .
\end{aligned}
$$

Then, each $v_{\kappa \mu \nu}\left(z^{\prime}\right)=u_{\nu}\left(\Phi_{\kappa \mu}\left(z^{\prime}\right)\right) \quad(\kappa, \mu, \nu=0,1,2, \cdots)$ is plurisubharmonic on $\Phi_{\kappa \mu}^{-1}\left(U_{\nu}\right)$, where $z^{\prime}=\left(z_{1}^{\prime}, \cdots, z_{n}^{\prime}\right)$. Since any set $F_{\kappa \mu \nu}:=\Phi_{\kappa \mu}^{-1}\left(U_{\nu}\right) \cap\left\{v_{\kappa \mu \nu}\left(z^{\prime}\right)=-\infty\right\}$ is of measure zero with respect to the coordinates $z^{\prime}$, we can find a point $(c, \underline{d}):=\left(c, d_{2}, \cdots, d_{n}\right)$ in $\boldsymbol{C} \times \boldsymbol{C}^{n-1}$ such that $0<|c|<1,\left|d_{i}\right|<1 \quad(2 \leqq i \leqq n)$ and $(c, \underline{d}) \notin F_{\kappa \mu \nu}$ for any $\kappa, \mu, \nu$ if $(c, \underline{d}) \in \Phi_{\kappa \mu}^{-1}\left(U_{\nu}\right)$. Consider the non-singular linear transformation

$$
\begin{gathered}
z_{1}=c w_{1} \\
z_{2}=d_{2} w_{1}+w_{2} \\
\ldots \\
z_{n}=d_{n} w_{1}+w_{n} .
\end{gathered}
$$

We have new local coordinates $w_{1}, w_{2}, \cdots, w_{n}$ which are well-defined on $W:=\left\{\left|w_{i}\right|<1 / 2,1 \leqq i \leqq n\right\}$. Let $\underline{w}:=\left(w_{2}, \cdots, w_{n}\right)$ and $w:=\left(w_{1}, \underline{w}\right)=$ $\left(w_{1}, \cdots, w_{n}\right)$. The functions $\tilde{u}_{\nu}\left(w_{1}, \underline{w}\right)=u_{\nu}\left(\Psi\left(w_{1}, \underline{w}\right)\right)$ defined on $W \cap \Psi^{-1}\left(U_{\nu}\right)$ satisfy the condition $\tilde{u}_{\nu}\left(a^{(\kappa)}, \underline{b}^{(\mu)}\right) \neq-\infty$ if $\left(a^{(\kappa)}, \underline{b}^{(\mu)}\right) \in \Psi^{-1}\left(U_{\nu}\right)$. So, $u_{\nu}\left(w_{1}, \underline{b}^{(\mu)}\right) \not \equiv-\infty$ on any connected component of $W \cap\left\{\underline{w}=\underline{b}^{(\mu)}\right\} \cap \Psi^{-1}\left(U_{\nu}\right)$ because $\left\{a^{(\kappa)}\right\}$ is dense in $\left\{\left|w_{1}\right|<1 / 2\right\}$. Since 


$$
W \cap S_{\nu} \cap\left\{\underline{w}=\underline{b}^{(\mu)}\right\} \subset\left\{u_{\nu}\left(w_{1}, \underline{b}^{(\mu)}\right)=-\infty\right\} \cap W \cap \Psi^{-1}\left(U_{\nu}\right)
$$

for any $\mu$ and $\nu$, it is considered as a set of capacity zero in the $w_{1}$-plane. In particular, $W \cap S \cap\left\{w_{2}=\cdots=w_{n}=0\right\}$ is of capacity zero in the $w_{1}$-plane. Then, as is well-known, there is an arbitrarily small real number $s_{1}$ such that

$$
\left(\left\{|w|=s_{1}\right\} \times\left\{w_{2}=\cdots=w_{n}=0\right\}\right) \cap S=\phi
$$

and so we can find real numbers $s_{1}^{\prime}, s_{1}^{\prime \prime}, s_{2}, \cdots, s_{n}$ such that $\left(\left\{s_{1}^{\prime} \leqq\left|w_{1}\right| \leqq s_{1}^{\prime \prime}\right\} \times\right.$ $\left.\left\{\left|w_{2}\right|<s_{2}, \cdots,\left|w_{n}\right|<s_{n}\right\}\right) \cap S=\phi$, where $0<s_{1}^{\prime}<s_{1}<s_{1}^{\prime \prime}<1 / 2$ and $0<s_{i}<$ $1 / 2(2 \leqq i \leqq n)$.

Put $U=\left\{\left|w_{i}\right|<s_{i}(1 \leqq i \leqq n)\right\}$. We want to prove that $f$ has a holomorphic continuation to $U$. It may be assumed that $f$ is bounded. Consider the function

$$
\tilde{f}\left(w_{1}, \underline{w}\right)=\frac{1}{2 \pi i} \int_{|\zeta|=s_{1}^{\prime \prime}} \frac{f(\zeta, \underline{w})}{\zeta-w_{1}} d \zeta
$$

Obviouly, it is holomorphic on $U$. On the other hand, each $h_{\mu}\left(w_{1}\right):=$ $f\left(w_{1}, b^{(\mu)}\right)$ is a bounded holomorphic function on $\left\{\left|w_{1}\right|<s_{1}\right\}$ except a closed set of capacity zero. It has exactly one holomorphic continuation to the whole $\left\{\left|w_{1}\right|<s_{1}\right\}$ (e.g. [8], p. 171), which ought to be equal to $\tilde{f}\left(w_{1}, \underline{b}^{(\mu)}\right)$. It follows that $f\left(w_{1}, \underline{b}^{(\mu)}\right)=\tilde{f}\left(w_{1}, \underline{b}^{(\mu)}\right)$ on $U-S$. Since $\left\{b^{(\mu)}\right\}$ is dense in $\left\{\left|w_{i}\right|<1 / 2,2 \leqq i \leqq n\right\}$, we conclude $f(w)=\tilde{f}(w)$ on the whole $U-S$. This shows that $\tilde{f}$ is a continuation of $f$ to $U$.

Now, we set about the proof of Proposition 3.1 for an arbitrary complex space $X$. By $X_{\text {reg }}$ we denote the set of all regular points of $X$. Then, it is considered as a complex manifold and $S \cap X_{\text {reg }}$ is a closed set of capacity zero in $X_{\text {reg. }}$. By the above proof, $f$ has a bounded holomorphic continuation to the whole $X_{\text {reg. }}$. Since $X-X_{\text {reg }}$ is a thin analytic subset of $X$, Proposition 3.1 is an immediate consequence of the well-known Riemann theorem on removable singularities of holomorphic functions on a normal complex space.

CoRollary 3.2. If $S$ is a closed set of capacity zero in a complex space, then $D-S$ is connected for any connected open subset $D$ of $X$.

Proof. Assume that $D-S$ is not connected, i.e. it can be written $D-S=D_{1} \cup D_{2}$ with mutually disjoint non-empty open sets $D_{1}$ and $D_{2}$. The bounded holomorphic function $f(z)$ on $D-S$ defined as $f(z)=i$ on $D_{i}$ 
$(i=1,2)$ is not continuable to $D$, which is contrary to the assumption by Proposition 3.1.

\section{$\S 4$. The boundary of a Riemann domain.}

By definition, a Riemann domain $(X, \pi, M)$ is a triple of complex spaces $X$ and $M$ with $\operatorname{dim} X=\operatorname{dim} M$ and a discrete holomorphic map $\pi: X \rightarrow M$. In this paper, the base space $M$ is always assumed to be a complex manifold.

Let $(X, \pi, M)$ be an arbitrary Riemann domain.

Definition 4.1. We shall say a sequence $\left\{x_{\nu}\right\}$ in $X$ to converge to the boundary of $X$ (relative to $M$ ) if $\left\{x_{\nu}\right\}$ has no accumulation point in $X$ and the sequence $\left\{\pi\left(x_{\nu}\right)\right\}$ has a limit $z_{0}$ in $M$. Such a point $z_{0}$, i.e. the limit of $\left\{\pi\left(x_{\nu}\right)\right\}$ for some $\left\{x_{\nu}\right\}$ converging to the boundary, is said a boundary value of $(X, \pi, M)$.

Definition 4.2. An accessible boundary point of $(X, \pi, M)$ is defined as a filter $r=\left\{U_{\iota} ; \iota \in I\right\}$ satisfying the conditions (i) there exist a point $z$ in $M$ and a fundamental system $\{V, ; \iota \in I\}$ of open connected neighborhoods of $z$ such that each $U_{c}$ is a connected component of $\pi^{-1}\left(V_{\imath}\right)$ and (ii) $U_{\imath} \in r$ is not relatively compact in $X$.

We denote the set of all accessible boundary points of $(X, \pi, M)$ by $\partial_{a} X$ and put $\check{X}=X \cup \partial_{a} X$. The set $\check{X}$ has a canonically defined locally connected Hausdorff topology such that a base for neighborhoods of each $r_{0}=\left\{U_{l}\right\} \in \partial_{a} X$ is given by the system $\left\{\check{U}_{t}\right\}$, where

$$
\check{U}_{\iota}:=U_{\iota} \cup\left\{r \in \partial_{a} X \text {; there exists some } V_{\kappa} \in r \text { with } V_{k} \subset U\right\} \text {. }
$$

The projection map $\pi$ is canonically extended to a continuous map $\pi: X \rightarrow M$. A sequence $\left\{x_{\nu}\right\}$ in $X$ converges to some $r \in \partial_{a} X$ if and only if $\left\{x_{\nu}\right\}$ converges to the boundary of $X$ and there is a continuous curve $r(t)(0<$ $t \leqq 1)$ in $X$ such that $\gamma(1 / \nu)=x_{\nu}$ for any $\nu$ and $\lim _{t \rightarrow 0} \pi(\gamma(t))=\lim _{\nu \rightarrow \infty} \pi\left(x_{\nu}\right)$.

Definition 4.3. An accessible boundary point $r \in \partial_{a} X$ is said to be a direct boundary point if there exists a neighborhood $U$ of $\check{\pi}(r)$ such that the connected component $U^{\prime}$ of $\pi^{-1}(U)$ which gives a neighborhood $\check{U}$ of $r$ satisfies the condition $U^{\prime} \cap \pi^{-1}(\check{\pi}(r))=\phi$.

By definition, we see easily

(4.4) For a point $r \in \partial_{a} X$, if $\pi^{-1}(\check{\pi}(r))$ contains only finitely many points in $X, r$ is a direct boundary point. 
Definition 4.5. A Riemann domain $(X, \pi, M)$ is said to have the boundary of capacity zero if each $z \in M$ has a neighborhood $U$ such that there exists a plurisubharmonic function $u(x)$ on $\pi^{-1}(U)$ with the following properties;

(i) $u(x) \leqq 0$,

(ii) $u(x) \not \equiv-\infty$ on any connected component of $\pi^{-1}(U)$,

(iii) $\lim _{\nu \rightarrow \infty} u\left(x_{\nu}\right)=-\infty$ for any sequence $\left\{x_{\nu}\right\}$ in $X$ which converges to the boundary.

Example 4.6. (i) Let $(X, \pi, M)$ be a Riemann domain with boundary of capacity zero and $D$ be a connected open subset of $M$. Then, for any connected component $D_{\iota}$ of $\pi^{-1}(D),\left(D_{\iota}, \pi \mid D_{\iota}, D\right)$ is also a Riemann domain with boundary of capacity zero.

(ii) If $X$ is a Riemann surface of type $O_{G}$ (e.g., see [10], p. 429) and $\psi(x)$ is a non-constant meromorphic function on $X$, a Riemann domain $(X, \psi, \boldsymbol{P})$ has the boundary of capacity zero, where $\boldsymbol{P}$ is the Riemann sphere.

For, if a Riemann surface $X$ is of type $O_{G}$, we can find a non-positive harmonic funcion $u(x)$ on $X-\bar{X}_{o}$ for an arbitrarily small suitable relatively compact open set $X_{o}$ in $X$ such that $\lim _{\nu \rightarrow \infty} u\left(x_{\nu}\right)=-\infty$ for any sequence $\left\{x_{\nu}\right\}$ in $X$ converging to the ideal boundary (c.f. M. Nakai [5], Theorem, p. 624).

(iii) Let $D$ be a domain in $C^{n}$ and $S$ be an at most $k$ dimensional analytic set in $D$. If $A$ is an irreducible analytic set in $D-S$ and $x$ is a point in $\bar{A} \cap S$, we can find polydiscs $U_{1}:=\left\{\left|z_{i}\right|<r_{i}, 1 \leqq i \leqq k\right\}, U_{2}:=$ $\left\{\left|z_{i}\right|<r_{i}, k+1 \leqq i \leqq n\right\} \quad\left(r_{i}>0\right)$ for a suitable system of local coordinates $z_{1}, \cdots, z_{n}$ on a neighborhood of $x$ with $x=(0)$ such that the Riemann domain $\left(X, \pi \cdot \mu, U_{1}\right)$ has the boundary of capacity zero, where $\pi:\left(z_{1}, \cdots, z_{n}\right)$ $\rightarrow\left(z_{1}, \cdots, z_{k}\right)$ is the canoncal projection and $X$ is the normalization of the locally analytic set $A \cap\left(U_{1} \times U_{2}\right)$ with projection map $\mu$.

To see this, we take a system of local coordinates $z_{1}, \cdots, z_{n}$ in a neighborhood $V$ of $x$ with $x=(0)$ such that the map $\pi:\left(z_{1}, \cdots, z_{n}\right) \rightarrow\left(z_{1}, \cdots, z_{k}\right)$ is discrete on $V \cap(A \cup S)$. Then we can find easily a sufficiently small poly$\operatorname{disc} U_{2}:=\left\{\left|z_{i}\right|<r_{i}, k+1 \leqq i \leqq n\right\}\left(r_{i}>0\right)$ with the property $\left(\left\{z_{1}=\cdots=\right.\right.$ $\left.\left.z_{k}=0\right\} \times \partial U_{2}\right) \cap(A \cup S)=\phi$ and hence a polydisc $U_{1}:=\left\{\left|z_{i}\right|<r_{i}, 1 \leqq i \leqq k\right\}$ $\left(r_{i}>0\right)$ such that $\left(U_{1} \times \partial U_{2}\right) \cap(A \cup S)=\phi$ because $A \cup S$ is closed, where $U:=U_{1} \times U_{2} \Subset V$. On the other hand, by the assumption we may assume 
that $U \cap S \subset\{f=0\}$ with a suitable holomorphic function $f(z)$ on $U$ which does not vanish identically on $A$. Using the plurisubharmonic function $u(x):=\log |(f \cdot \mu)(x)|$ on $X$, we can easily conclude that these polydiscs $U_{1}$ and $U_{2}$ satisfy the desired condition.

\section{$\S 5$. The projection image of a Riemann domain with boundary of capacity zero.}

The following theorem is a generalization of a result in [4] (c.f. Tsuji, [10]. p. 437) to the case of Riemann domains of arbitrary dimension.

Theorem 5.1. If $(X, \pi, M)$ is a Riemann domain with boundary of capacity zero, then $M-\pi(X)$ is a closed set of capacity zero in $M$.

For the proof, we give the following

Lemma 5.2. Let $(X, \pi, M)$ be a Riemann domain such that $\pi$ is proper and $u(x)$ be a plurisubharmonic function on $X$. If we put

$$
w(z):=\max \{u(x) ; \pi(x)=z, x \in X\}
$$

for each $z \in M$, then the function $w(z)$ is plurisubharmonic on $M$.

Proof. By the assumption, there is a thin analytic set $N$ in $M$ such that $\left(X-\pi^{-1}(N), \pi \mid X-\pi^{-1}(N), M-N\right)$ is an unramified proper covering space. If we put $\pi^{-1}(z)=\left\{x_{1}(z), \cdots, x_{m}(z)\right\} \quad(z \in M-N)$, then $w(z)=\max _{1 \leqq i \leqq m} u\left(x_{i}(z)\right)$ is plurisubharmonic on $M-N$ by (2.2). To prove Lemma 5.2 , it suffices to show $\varlimsup_{z \rightarrow z_{o},} \varlimsup_{z \in M-N} w(z)=w\left(z_{o}\right)$ for any $z_{o} \in N$ because of (2.3).

Take an arbitrary sequence $\left\{z_{\nu}\right\}$ in $M-N$ such that $\lim _{\nu \rightarrow \infty} z_{\nu}=z_{0}\left(z_{0} \in N\right)$ and $\lim _{\nu \rightarrow \infty} w\left(z_{\nu}\right)=\varlimsup_{z \rightarrow z_{0},} \varlimsup_{z \in M-N} w(z)$. By the definition of $w(z)$, there is a sequence $\left\{x_{\nu}\right\}$ with the properties $\pi\left(x_{\nu}\right)=z_{\nu}$ and $w\left(z_{\nu}\right)=u\left(x_{\nu}\right)$, which may be assumed to have a limit point $x_{0}$ in $X$ by the properness of $\pi$. Then we get

$$
\varlimsup_{z \rightarrow z_{o},} \varlimsup_{z \in M-N} w(z)=\lim _{\nu \rightarrow \infty} u\left(x_{\nu}\right) \leqq \varlimsup_{x \rightarrow x_{o}} u(x) \leqq w\left(z_{o}\right) .
$$

On the other hand, if we take a point $x_{o} \in X$ such that $w\left(z_{o}\right)=u\left(x_{o}\right)$ and $\pi\left(x_{0}\right)=z_{0}$, there is a sequence $\left\{x_{\nu}\right\}$ in $X-\pi^{-1}(N)$ satisfying the condition $\lim _{\nu \rightarrow \infty} x_{\nu}=x_{0}$ and $\lim _{\nu \rightarrow \infty} u\left(x_{\nu}\right)=u\left(x_{0}\right)$ because of $(2.4)$. Hence we have

$$
w\left(z_{0}\right)=\lim _{\nu \rightarrow \infty} u\left(x_{\nu}\right) \leqq \lim _{\nu \rightarrow \infty} w\left(\pi\left(x_{\nu}\right)\right) \leqq \varlimsup_{z \rightarrow z_{0},} \varlimsup_{z \in M-N} w(z) .
$$


This completes the proof.

Lemma 5.3. Let $u(x)$ be a plurisubharmonic function on a Riemann domain $(X, \pi, M)$ such that (i) $0 \geqq u(x) \not \equiv-\infty$ and (ii) $\lim _{\nu \rightarrow \infty} u\left(x_{\nu}\right)=-\infty$ for any sequence $\left\{x_{\nu}\right\}$ in $X$ which converges to the boundary. If we put

$$
w(z)= \begin{cases}\sup \{u(x) ; \pi(x)=z, x \in X\} & \text { for any } z \in \pi(X) \\ -\infty & \text { for any } z \notin \pi(X),\end{cases}
$$

it is plurisubharmonic on $M$.

Proof. Firstly, we shall show that $w(z)$ is upper semicontinuous on $M$. For any $z_{0} \in M$, take a sequence $\left\{z_{\nu}\right\}$ in $M$ such that $\lim _{\nu \rightarrow \infty} z_{\nu}=z_{0}$ and $K:=\lim _{\nu \rightarrow \infty} w\left(z_{\nu}\right)$ exists. We want to prove $K \leqq w\left(z_{0}\right)$. Let $K \neq-\infty$ because, if not, the proof is trivial. Choosing a subsequence and changing indices if necessary, we may assume that there is a sequence $\left\{x_{\nu}\right\}$ in $X$ such that $\pi\left(x_{\nu}\right)=z_{\nu}, \lim _{\nu \rightarrow \infty} u\left(x_{\nu}\right)=K$ and, moreover, $\left\{x_{\nu}\right\}$ has a limit $x_{0}$ in $X$ by the assumption (iii) in Lemma 5.3. Since $\pi\left(x_{0}\right)=z_{0}$ and $u(x)$ is upper semicontinuous, it holds that

$$
K=\lim _{\nu \rightarrow \infty} u\left(x_{\nu}\right) \leqq \varlimsup_{x \rightarrow x_{o}} u(x) \leqq u\left(x_{o}\right) \leqq w\left(z_{o}\right) .
$$

This shows that $w(z)$ is upper semi-continuous.

To complete the proof, taking an open set $W$ in $C$ and a holomorphic $\operatorname{map} \psi: W \rightarrow M$, we shall prove that

$$
(w \cdot \psi)\left(t_{o}\right) \leqq \frac{1}{2 \pi} \int_{0}^{2 \pi}(w \cdot \psi)\left(t_{o}+r \mathrm{e}^{i \theta}\right) d \theta
$$

for any $t_{0} \in W$ and a sufficiently small arbitrary positive real number $r$. We may assume $(w \cdot \psi)\left(t_{0}\right) \neq-\infty$. Consider the set $E:=\left\{x \in X ; u(x)=w\left(z_{o}\right)\right.$, $\pi(x)=z_{o}$, where $z_{o}=\pi\left(t_{o}\right)$. By the properties of $\pi$ and $u, E$ is a finite set. We can find easily neighborhoods $V$ of $E$ and $U$ of $z_{0}$ such that the map $\pi^{\prime}:=\pi \mid V: V \rightarrow U$ is proper. Then the Riemann domain $\left(V, \pi^{\prime}, U\right)$ satisfies the assumption in Lemma 5.2. So, the function $\tilde{w}(z):=\max \{u(x)$; $\pi(x)=z, x \in V\}$ is plurisubharmonic and, obviously, satisfies the conditions $\tilde{w}\left(z_{o}\right)=w\left(z_{o}\right)$ and $\tilde{w}(z) \leqq w(z)$ on $U$. We obtain

$$
\begin{aligned}
w\left(z_{o}\right) & =(\tilde{w} \cdot \phi)\left(t_{o}\right) \leqq \frac{1}{2 \pi} \int_{0}^{2 \pi}(\tilde{w} \cdot \psi)\left(t_{o}+r \mathrm{e}^{i \theta}\right) d \theta \\
& \leqq \frac{1}{2 \pi} \int_{0}^{2 \pi}(w \cdot \psi)\left(t_{o}+r \mathrm{e}^{i \theta}\right) d \theta
\end{aligned}
$$


for any sufficiently small $r>0$. This asserts that $w(z)$ is plurisubharmonic on $M$.

Proof of Theorem 5.1. Let $z_{o}$ be an arbitrary point in the boundary $\partial \pi(X)$ of $\pi(X)$. By the assumption, for a suitable neighborhood $U$ of $z_{o}$, there is a plurisubharmonic function $u(x)$ on $\pi^{-1}(U)$ satisfying the conditions in Definition 4.5, (i) (iii). For an arbitrarily fixed connected component $V$, of $\pi^{-1}(U)$, the Riemann domain $\left(V_{\imath}, \pi \mid V_{\imath}, U\right)$ satisfies the assumption in Lemma 5.3. The function

$$
w(z):= \begin{cases}\sup \left\{u(x) ; \pi(x)=z, x \in V_{\imath}\right\} & \text { for any } z \in \pi\left(V_{\iota}\right) \\ -\infty & \text { for any } z \in U-\pi\left(V_{\iota}\right)\end{cases}
$$

is plurisubharmonic on $U$. Obviously, $U \cap \partial \pi(X)=\{z \in U ; w(z)=-\infty\}$, which is of capacity zero. Since $\partial \pi(X)$ is covered by countably many $U^{\prime} \mathrm{s}$ with the above properties, it is of capacity zero. Then we have $M-\pi(X)=\partial \pi(X)$ and hence Theorem 5.1. Indeed, if not, $M-\pi(X)$ has a boundary point in $M-\partial \pi(X)$ because $M-\partial \pi(X)$ is connected by Corollary 3.2, which is absurd.

\section{$\S 6$. Direct boundary points of Riemann domains with boundary of capacity zero.}

Using Theorem 5.1, we can prove the following theorem on direct boundary points of a Riemann domain, which is a generalization of $\mathrm{A}$. Mori [4], Corollary 2, p. 288.

Theorem 6.. If $(X, \pi, M)$ is a Riemann domain with boundary of capacity zero, the projection image of the set of all direct boundary points of $X$ is a set of capacity zero in $M$.

Proof. Let $\mathfrak{A}=\left\{U_{\nu}\right\}$ be a countable base for connected open sets in $M$. For each $U_{\nu}$, each connectd component $U_{\nu}^{\iota}$ of $\pi^{-1}\left(U_{\nu}\right)$ defines a Riemann domain $\left(U_{\nu}^{i}, \pi \mid U_{\nu}^{i}, U_{\nu}\right)$ which has the boundary of capacity zero. According to Theorem 5.1, the set $F_{\nu}^{i}:=U_{\nu}-\pi\left(U_{\nu}^{i}\right)$ is of capacity zero in $U_{\nu}$. So, $F:=\cup_{\iota, \nu} F_{\nu}$ is also of capacity zero in $M$. We need only to show that the $\check{\pi}$-image of an arbitrary direct boundary point of $(X, \pi, M)$ is contained in $F$. Let $r \in \partial_{a} X$ be an arbitrary direct boundary point and put $\check{\pi}(r)=z$. By definition, there is a neighborhood $U$ of $z$ such that a connected component $U^{\prime}$ of $\pi^{-1}(U)$ with $U^{\prime} \in r$ satisfies the condition $U^{\prime} \cap \pi^{-1}(\pi(r))=\phi$, where we 
may assume $U=U_{\nu_{0}} \in \mathfrak{A}$ and $U^{\prime}=U_{\nu_{0}^{\prime}}^{\iota_{0}}$ for some $\nu_{0}$ and $\iota_{0}$. Obviously, $z \in F_{\nu_{0}}^{\mathrm{c}_{0}} \subset F$. This completes the proof.

As an application of Theorem 6.1, we can generalize the result of $\mathrm{W}$. Rothstein [8], p. 172 to the case of Riemann domains of arbitrary dimension.

Corollary 6.2. Assume that $(X, \pi, M)$ has the boundary of capacity zero. If $P$ is a subset of the set of all boundary values of $(X, \pi, M)$ such that $\pi^{-1}(c)$ is finite for any $c \in P$, it is of capacity zero.

Proof. Assume that cap $P>0$. Without loss of generality, we may assume that $P$ contains no direct boundary point because of Theorem 6.1. Consider the set

$$
P_{\nu}=\left\{z \in P ; \pi^{-1}(z) \text { consists of at most } \nu \text { points }\right\}
$$

for any $\nu=0,1,2, \cdots$. Since $P=\cup_{\nu} P_{\nu}$, there is some $\nu_{0}$ such that cap $P_{\nu_{0}}>0$ by (2.6), (i). Here, it cannot happen to be $\nu_{0}=0$ in virtue of Theorem 5.1. Moreover, according to $(2.6)$ (iii),

$$
P^{\prime}:=\left\{z \in P_{\nu_{0}} ; \operatorname{cap}\left(P_{\nu_{0}} \cap U\right)>0 \text { for any neighborhood } U \text { of } z\right\}
$$

is of positive capacity. Take a point $z_{o}$ in $P^{\prime}$ and an arbitrary neighborhood $U$ of $z_{o}$. For any connected component $U_{\iota}$ of $\pi^{-1}(U)$, since $\operatorname{cap}\left(U-\pi\left(U_{\imath}\right)\right)=0$, we can choose a point $z_{1} \in P^{\prime} \cap U$ such that $z_{1} \notin U_{\iota}\left(U-\pi\left(U_{\imath}\right)\right)$, whence $z_{1} \in \cap \pi\left(U_{\imath}\right)$. This shows that $\pi^{-1}(U)$ has at most $\nu_{0}$ connected components. Since $z_{o}$ is the boundary value of $(X, \pi, M)$, there is at least one connected component of $\pi^{-1}(U)$ which is not relatively compact in $X$. This concludes that $z_{o}$ is the $\check{\pi}$-image of an accessible boundary point of $(X, \pi, M)$, which is absurd because of (4.4). Hence Corollary 6.2 is proved.

The following theorem is essentially another description of the Bishop's result in [1], Theorem 4, p. 301 (c.f. Theorem 7.3 in the following section).

Theorem 6.3. Let $(X, \pi, M)$ be a Riemann domain with boundary of capacity zero. If there is at least one point in $M$ which is not a boundary value of $(X, \pi, M)$, then $\pi^{-1}(z)$ is finite for any $z \in M$ and, moreover, there exists a closed set $N$ of capa. city zero in $M$ such that $\pi \mid X-\pi^{-1}(N): X-\pi^{-1}(N) \rightarrow M-N$ is proper.

Proof. Since the set $S$ of all boundary values is closed, we can take a connected open set $U$ in $M$ such that $U \cap S=\phi$. Obviously, the map $\pi \mid \pi^{-1}(U): \pi^{-1}(U) \rightarrow U$ is proper. Consider the set $G$ of all points $z$ in $M$ 
with the property that $\pi \mid \pi^{-1}(V): \pi^{-1}(V) \rightarrow V$ is proper for some neighborhood $V$ of $z$. Then obviously, $\pi \mid \pi^{-1}(G): \pi^{-1}(G) \rightarrow G$ itself is also proper and $\partial G \subset S$. Moreover, $\pi^{-1}(z)$ is finite for any $z \in \partial G$. In view of Corollary $6.2, \partial G$ is of capacity zero and so $N:=M-G=\partial G$ as in the proof of Theorem 5.1. This concludes Theorem 6.3.

Corollary 6.4. For a Riemann domain $(X, \pi, M)$ with boundary of capacity zero, if there is a set $P$ in $M$ such that cap $P>0$ and $\pi^{-1}(z)$ is a finite set for any $z \in P$, the same conclusion in Theorem 6.3 is valid (c.f. W. Rothstein [8], Satz 1, p. 173).

The proof is evident by Corollary 6.2 and Theorem 6.3.

Now, we give another application of Theorem 5.1. The following is a generalization of the well-known Iversen's theorem.

Theorem 6.5. Assume that $(X, \pi, M)$ has the boundary of capacity zero. Take an arbitrary point $z_{0}$ in $M$ and a connected neighborhood $U$ of $z_{0}$. Then, for any $x_{0} \in \pi^{-1}(U)$, there exists a continuous curve $\gamma(t) \quad(0<t \leqq 1)$ in $\pi^{-1}(U)$ such that $\gamma(1)=x_{0}$ and $\lim _{t \rightarrow 0}(\pi \cdot \gamma)(t)=z_{0}$.

Proof. Let $\left\{U_{\nu}\right\}$ be a countable base for connected open neighborhoods of $z_{0}$. It suffices to show that there is a sequence $\left\{x_{\nu}\right\}$ such that $x_{\nu} \in \pi^{-1}\left(U_{\nu}\right)$ and a suitable continuous curve $\gamma_{\nu}(t)$ in $\pi^{-1}\left(U_{\nu}\right)$ joins $x_{\nu}$ with $x_{\nu+1}$ for any $\nu$. We proceed by induction on $\nu$. Assume that there exist points $x_{\mu}$ and curves $\gamma_{\mu}(1 \leqq \mu \leqq \nu)$ with the desired properties. Let $U^{\prime}$ be a connected component of $\pi^{-1}\left(U_{\nu}\right)$ which contains $x_{\nu}$. Since $\left(U^{\prime}, \pi \mid U^{\prime}, U_{\nu}\right)$ has the boundary of capacity zero, we see $\operatorname{cap}\left(U_{\nu}-\pi\left(U^{\prime}\right)\right)=0$. If we choose an arbitrary $x_{\nu+1} \in U^{\prime}$ with $\pi\left(x_{\nu+1}\right) \in U_{\nu+1}, x_{\nu}$ is joined with $x_{\nu+1}$ by a continuous curve $\gamma_{\nu+1}$ in $U^{\prime}$. These $x_{\nu+1}$ and $\gamma_{\nu+1}$ are the desired ones.

\section{§7. Continuations of Riemann domains and analytic sets.}

Applying the results of the previous sections, we can give some sufficient conditions for the continuability of Riemann domains and analytic sets by the similar arguments as in W. Rothstein [8].

Theorem 7.1. Let $(X, \pi, M)$ be a Riemann domain with boundary of capacity zero. Assume that there is a set $P$ in $M$ with the properties that (i) cap $P>0$, (ii) for any $z \in P \pi^{-1}(z)$ is finite and (iii) for each $z_{o} \in P$ a suitable bounded holo- 
morphic function $f$ on $X$ satisfies the condition $f\left(x_{i}\right) \neq f\left(x_{j}\right)(i \neq j)$, where $\pi^{-1}\left(z_{0}\right)=\left\{x_{1}, \cdots, x_{m}\right\}$. Then the space $\check{X}=X \cup \partial_{a} X$ defined as in $\S 4$ has a structure of a complex space such that $X$ is an open subspace of $\check{X}$ with $\operatorname{cap}(\check{X}-X)=0$ and the projection $\check{\pi}$ is a proper discrete holomorphic map.

For the proof, we need

Lemma 7.2. For a Riemann domain $(X, \pi, M)$, if it has the boundary of capacity zero and $\pi^{-1}(z)$ contains at most finitely many points for any $z \in M$, it holds that (i) $X$ is dense in $\check{X}$, (ii) $D \cap X$ is connected for any connected open subset $D$ of $\check{X}$, (iii) $\check{X}$ is locally compact and (iv) $\check{\pi}: \check{X} \rightarrow M$ is proper discrete.

Proof. The properties (i) and (ii) are evident by the definition of the topology of $\check{X}$ and (iii) follows from (iv). It remains only to prove (iv). As is easily seen, there is an integer $\nu_{0}(>0)$ such that $\pi^{-1}(z)$ consists of at most $\nu_{0}$ points for any $z \in M$. Moreover, as in the proof of Corollary 6.2, $\pi^{-1}(U)$ has at most $\nu_{o}$ connected components for any connected open set $U$ in $M$. This implies that any sequence converging to the boundary has a subsequence converging to an accessible boundary point and so $\pi$ is proper. The discreteness of $\check{\pi}$ is obvious because $\check{\pi}^{-1}(z)(z \in M)$ contains at most $\nu_{0}$ points.

Proof of Theorem 7.1. By Corollary 6.4, $\pi \mid X-\pi^{-1}(N): X-\pi^{-1}(N) \rightarrow M-N$ is proper for a suitable closed set $N$ of capacity zero in $M$, where $\left(X-\pi^{-1}(N)\right.$, $\left.\pi \mid X-\pi^{-1}(N), M-N\right)$ may be assumed to be an unramified proper covering space. Put $X^{\prime}:=X-\pi^{-1}(N)$ and $\pi^{\prime}:=\pi \mid X-\pi^{-1}(N)$. Since $\operatorname{cap}\left(\pi^{-1}(N)\right)=0$, we see easily $\check{X}^{\prime}=\check{X}$ as topological spaces and $\check{\pi}^{\prime}=\check{\pi}$, where $\check{X}^{\prime}=X \cup \partial_{a} X^{\prime}$ and $\check{\pi}^{\prime}$ is an extension of $\pi^{\prime}$ to $\check{X}^{\prime}$ as defined in $\S 4$ for a Riemann domain $\left(X^{\prime}, \pi^{\prime}, M\right)$. There is no harm in assuming that $X=X^{\prime}$ and $\pi=\pi^{\prime}$.

Put $\pi^{-1}(z)=\left\{x_{1}(z), \cdots, x_{m}(z)\right\}$ for any $z \in M-N$. Using the holomorphic function $f$ on $X$ given for some $z_{o} \in P-N$, we define the pseudopolynomial

$$
\Pi_{i=1}^{m}\left(w-f\left(x_{i}(z)\right)\right)=w^{m}+a_{1}(z) w^{m-1}+\cdots+a_{m}(z)
$$

whose coefficients $a_{i}(z)$ are holomorphic on $M-N$ and locally bounded on $N$. By virtue of Proposition 3.1, each $a_{i}(z)$ has a holomorphic continuation $\tilde{a}_{i}(z)$ to the whole $M$. Let $Y$ be the analytic set in $M \times C$ defined by the equation 


$$
w^{m}+\tilde{a}_{1}(z) w^{m-1}+\cdots+\tilde{a}_{m}(z)=0
$$

and take the normalization $\mu: \tilde{Y} \rightarrow Y$ of $Y$. For the canonical projection $\pi_{1}:(z, w) \rightarrow z$, putting $\tilde{\pi}=\pi_{1} \cdot \mu$, we have proper finite covering space $(\tilde{Y}, \tilde{\pi}, M)$. By the assumption of $f, \tilde{\pi}^{-1}(z)$ contains exactly $m$ points for any $z \in M$ except a thin analytic set. Then there is a homeomorphism $\tau$ of $\tilde{Y}-\tilde{\pi}^{-1}(N)$ onto $X-\pi^{-1}(N)$ such that $\pi \tau=\tilde{\pi}$ on $\tilde{Y}-\tilde{\pi}^{-1}(N)$. In this situation, by virtue of Lemma 7.2 , we can easily prove that $\tau$ has an extension $\tilde{\tau}: \tilde{Y} \rightarrow \check{X}$ with $\check{\pi} \tilde{\tau}=\tilde{\pi}$ which gives a homeomorphism between $\tilde{Y}$ and $\check{X}$ by the analogous argument as in the proof of the uniqueness of the normalization of a not necessarily normal reduced complx space (c.f. [3], Satz 2, p. 250). We can define a structure of a complex space on $\check{X}$ such that $\tilde{\tau}$ is biholomorphic. The Riemann domain $(X, \tilde{\pi}, M)$ obtained in this manner satisfies obviously the conditions in Theorem 7.1.

Now, we shall prove Theorem III stated in $\S 1$ under slightly weaker assumptions.

Theorem 7.3. Let $M$ be a complex manifold of dimension $n$. Assume that an irreducible $n$-dimensional analytic set $A$ in some open subset of $M \times \boldsymbol{C}^{k}$ satisfies the following conditions;

(i) there is a plurisubharmonic function $u(x)$ on $A$ such that $0 \geqq u(x) \not \equiv-\infty$ and $\lim _{\nu \rightarrow \infty} u\left(x_{\nu}\right)=-\infty$ for any sequence $\left\{x_{\nu}\right\}$ in $A$ without accumulation points if $\lim _{\nu \rightarrow \infty} \pi_{1}\left(x_{\nu}\right)$ exists,

(ii) $\pi_{2}(A)$ is a bounded subset of $\boldsymbol{C}^{k}$,

(iii) there is a set $P$ in $M$ such that $\operatorname{cap}(P)>0$ and $\pi_{1}^{-1}(z) \cap A$ is finite for any $z \in P$,

where $\pi_{1}: M \times C^{k} \rightarrow M$ and $\pi_{2}: M \times C^{k} \rightarrow C^{k}$ are the canonical projections.

Then, $\bar{A}$ is analytic in $M \times \boldsymbol{C}^{k}$.

Proof. Let $\mu: X \rightarrow A$ be the normalization of $A$ and put $\pi:=\pi_{1} \cdot \mu$. The Riemann domain $(X, \pi, M)$ has the boundary of capacity zero. By Corollary 6.4, $\pi \mid X-\pi^{-1}(N): X-\pi^{-1}(N) \rightarrow M-N$ is proper if we take a suitable closed subset $N$ of capacity zero in $M$. Then, $\pi_{1} \mid A-\pi_{1}^{-1}(N)$ : $A \rightarrow \pi_{1}^{-1}(N) \rightarrow M-N$ is also proper. As usual, for each coordinate $w_{i}(1 \leqq i \leqq k)$ in $\boldsymbol{C}^{k}$, we take the equation 


$$
P_{i}\left(z ; w_{i}\right)=w_{i}^{m}+a_{1}^{(i)}(z) w_{i}^{m-1}+\cdots+a_{m}^{(i)}(z)=0
$$

on $A-\pi_{1}^{-1}(N)$, where $a_{j}^{(i)}$ are bounded holomorphic on $M-N$ and so have holomorphic continuations $\tilde{a}_{j}^{(i)}$ to $M$. If we take the analytic set $A^{\prime}:=$ $\left\{w_{i}^{m}+\tilde{a}_{1}^{(i)} w_{i}^{m-1}+\cdots+\tilde{a}_{m}^{(i)}=0,1 \leqq i \leqq k\right\}$, it can be easily seen that the irreducible component of $A^{\prime}$ including $A \cap\left((M-N) \times C^{k}\right)$ coincides with the set $\bar{A}$. This shows that $\bar{A}$ is analytic in $M \times C^{k}$.

Lastly we note that the Thullen-Remmert-Stein's theorem on the essential singularities of analytic sets in [9] and [7] is an immediate consequence of Theorem 7.3 and Example 4.6, (iii).

\section{REFERENCES}

[ 1] E. Bishop, Conditions for the analyticity of certain sets, Mich. Math. J., 11 (1964), 289304.

[2] H. Grauert and R. Remmert, Plurisubharmonisches Funktionen in komplexen Räume, Math. Z., 65 (1956), 175-194.

[3] H. Grauert and R. Remmert, Komplexe Räume, Math. Ann., 136 (1958), 245-318.

[4] A. Mori, On Riemann surfaces, on which no bounded harmonic function exists, J. Math. Soc. Japan, 3 (1951), 285-289.

[5] M. Nakai, On Evans potential, Proc. Japan Acad., 38 (1962), 624-629.

[6] T. Nishino, Sur les ensembles pseudoconcaves, J. Màth. Kyoto Univ., 1 (1962), 225-245.

[ 7 ] R. Remmert and K. Stein, Über die wesentlichen singularitäten analytischer Mengen, Math. Ann., 126 (1953), 263-306.

[8] W. Rothstein, Das Maximumprinzip und die Singularitäten analytischer Mengen, Inv. Math., 6 (1968), 163-184.

[9] P. Thullen, Über die wesentlichen Singularitäten analytischer Funktionen und Flächen im Räume von n komplexen Veränderlichen, Math. Ann., 111 (1935), 137-157.

[10] M. Tsuji, Potential theory in modern function theory, Maruzen, Tokyo, 1959. 